\title{
Review of Clinical Features and Treatment of Post-Traumatic Stress Disorder in Children
}

\author{
Lufei Lin*, Tian Liu, Yu Zheng, Yuebiao Wu, Dingsheng Liang, Ming Wu, Jianguo Li, Xinjian Mo, \\ Hualan Lin, Xueyin Jiang
}

The Fourth People's Hospital of Haikou, Haikou, China

Email: ^g2002m@163.com

How to cite this paper: Lin, L.F., Liu, T., Zheng, Y., Wu, Y.B., Liang, D.S., Wu, M., Li, J.G., Mo, X.J., Lin, H.L. and Jiang, X.Y. (2022) Review of Clinical Features and Treatment of Post-Traumatic Stress Disorder in Children. Journal of Behavioral and Brain Science, 12, 1-9.

https://doi.org/10.4236/jbbs.2022.121001

Received: December 4, 2021

Accepted: January 10, 2022

Published: January 13, 2022

Copyright $\odot 2022$ by author(s) and Scientific Research Publishing Inc. This work is licensed under the Creative Commons Attribution International License (CC BY 4.0).

http://creativecommons.org/licenses/by/4.0/

\begin{abstract}
Childhood is an important period for personality formation, cognition and social development. Childhood physical and mental trauma is the key reason for the development of many serious diseases. Severe traumatic events can cause Post-Traumatic Stress Disorder (PTSD). Children's psychological adjustment and tolerance are generally lower than adults, and it is difficult for children to seek reasonable help after suffering traumatic events. Children who experience a traumatic event have an even higher rate of PTSD than adults, considering problems with their symptom presentation. Post-Traumatic Stress Disorder can hinder children's development of healthy psychology such as independence and autonomy in the future, and can lead to a series of psychological disorders in adulthood. This article introduces the epidemiology, symptoms, physiological changes and influencing factors of PTSD in children, and puts forward the direction of treatment in the future.
\end{abstract}

\section{Keywords}

Children, Post-Traumatic Stress Disorder, Physiology, Review

\section{Introduction}

Post-Traumatic Stress Disorder (PTSD) is a typical psychological stress disease. Post-Traumatic Stress Disorder (PTSD) is a post-traumatic psychological disorder that is a delayed and/or prolonged anxious response to an unusually threatening or catastrophic event. PTSD refers to the severe anxiety disorder that occurs after the trauma such as earthquake, fire and domestic violence. Its high incidence and long course seriously affect the prognosis of the trauma, thus triggering a series of physical, psychological and behavioral stress responses. Acute and intense trauma can lead to physical and mental sequelae, severe physical deformity and 
even obvious mental trauma in affected individuals, especially in children. The disease has been an independent disease unit for only 30 years. Like many other diseases, research on childhood PTSD lags far behind that of adults, so understanding of it is even more limited. Data [1] show that the treatment of post-traumatic PTSD in children becomes more difficult once the symptoms are not timely diagnosed and treated. Therefore, early detection of PTSD in children is particularly important for the physical and psychological maturity of children and adolescents. This article reviews the epidemiology, symptoms, physiological changes and the treatment of PTSD in children.

\section{Epidemiology}

The lifetime prevalence of PTSD in the general population is about $1 \%-14 \%$ [2]. Giaconia et al. [3] reported that in a community survey, more than $2 / 5$ young people under the age of 18 had experienced at least one traumatic event in accordance with the PTSD criteria of DSM-iii-R, and more than $6 \%$ met the diagnosis of PTSD. Cuffe et al. [4] reported that among young people aged 16 - 22 in one community, $1 \%$ of males and $3 \%$ of females met the CRITERIA of DSM-IV PTSD.

\section{The Causes of PTSD in Children}

Ball et al. [5] believed that re-exposure to the scene, people and objects similar to the time of injury may be an important factor causing children's PTSD. In addition, parents' reaction to traumatic events and children's post-traumatic illness is also an important indicator of the occurrence and severity of children's PTSD [6] [7]. Studies have shown that the more intensely a child's guardian reacts to a traumatic event and the more anxious he or she is about the child's post-traumatic condition, the higher the incidence of PTSD in children is. In addition, individual differences of children also have a great impact on the development of PTSD. Children with anxiety personality traits are more likely to develop PTSD, and children with higher IQ are more tolerant to trauma than children with normal and low IQ as measured by psychological scale [8]. Dana et al. [9] in the study also found that children PTSD is not only appear after a major disaster or all kinds of accidents, also can appear in all kinds of invasive operations, such as suffering from congenital heart disease children's heart surgery, also can lead to PTSD symptoms appear, and that the incidence of PTSD symptoms and children within the TCU for time has a positive correlation. Young et al. [10] reported that $5 \%$ of children with leukemia who underwent bone marrow transplantation developed PTSD symptoms of varying degrees within 12 months after surgery. In addition, organ transplantation, domestic violence and even chronic diseases may be the causes of children's PTSD [11] [12] [13].

\section{Physiological and Biochemical Changes}

Psychological trauma can cause neurophysiological and neurobiological changes. 
Henschel et al. [14] emphasized that the "fight or flight" response pattern of adults is rarely suitable for young children. Young children initially respond to trauma with increased alertness. This is a signal to the caregiver to ask for attention and help. But without help, as the trauma or threat continues, the child's next response is to fix or "solidify," and then separate or "surrender." Physiological responses such as heart rate can be normalized when children begin to develop separation symptoms. It has been reported that the normal inhibitory regulation of the shock response has disappeared in children. Glod et al. [15] found differences in circadian rhythms and activity levels between abused children with and without PTSD, and the former was similar to attention deficit hyperactivity disorder. It is also believed that a marked increase in neurotransmitter activity associated with severe and persistent stress can affect brain development in children and is therefore considered a risk factor for developmental disorders. A study of adolescents five years after an earthquake found a link between intrusive symptoms and basal cortisol levels and cortisol suppression by dexamethasone.

\section{Symptoms of PTSD in Children}

Generally speaking, the core symptoms of PTSD fall into three groups: re-experience or intrusion symptoms (flashbacks, intrusive memories, nightmares, etc.); Avoidance or numbness symptoms (avoidance of trash-related stimuli, sensations and activities, etc.) and hyperarousal symptoms (anxiety, sleep disturbance, anger, irritability and excessive startled response), among which the avoidance or numbness symptoms lasted the longest [16] [17]. It should be noted that the clinical manifestations of PTSD in children are not exactly the same as those in adults. The main manifestations are fear of separation from parents, forgetting certain skills acquired in the past, frequent sleep disturbances and recurrent nightmares, depression and obsessive-compulsive symptoms, etc. What's interesting is that PTSD varies across age groups. Most children show avoidance symptoms after traumatic events, reexperience and hyperarousal are more common in adolescents. Winston [18] research points out that children may by repeating certain behavior or action to avoid breaking into sexual symptoms appear, children should be alert to the occurrence of PTSD, clinicians need to closely observe clinical manifestations of children and timely intervention, otherwise once appear PTSD, will be on the mental health development of children with serious negative impact. Although previous studies suggested that burns played little role in the occurrence of PTSD in children [19] [20], Meyer et al. [21] found in a clinical study of children with extensive burns (mean TBSA $=48 \%$ ) with an average age of 3.3 years. The physical and psychological status of most of the children has changed significantly after burn, and these children have different degrees of social function regression, depression, anxiety and other psychological states. Through further studies, Johnson et al. [22] found that various functions of children with burns were reduced to a certain extent, especially their language expression ability was significantly inferior to that of normal children of the same age. Subsequent 
investigations also found that burn area, pain degree and parents' acute stress state after traumatic events were closely related to the incidence of PTSD and severity of symptoms [23]. These results strongly suggest that burn plays an important role in the development of PTSD in children, and that early physical and psychological intervention is an effective means to prevent PTSD and improve the prognosis of burn children.

\section{Clinical Manifestations and Course of Disease}

The basic features of PTSD are three characteristic clusters of symptoms following traumatic events: 1) persistent re-experience of stressors; 2) Persistent avoidance of traumatic memories and numbness of general responses; 3 ) Persistent vigilance symptoms. Children may present with repeated play about traumatic events, generalized nightmares, psychosomatic symptoms and their precursors, causing distress or dysfunction that lasts for more than a month. Symptoms usually begin within three months of the trauma, but can be delayed for months or even years. There may be some behavioral block or enhancement after trauma, especially in the early stages of trauma. Symptoms change with time, most of which gradually decrease [24] [25], while a few persist or even get worse. Children's symptoms are often incomplete and may not fully meet diagnostic criteria. In some cases, the full cluster of symptoms appears late, and children's PTSD is often chronic, which can impair development. Therefore, it is important to inquire about all three symptom groups and to consider treatment when the symptoms do not fully meet the diagnostic criteria.

\section{Influencing Factors and Particularity of Children}

Research shows that children PTSD bad personality traits associated with larger, and the evolution of this kind of personality traits are closely associated with unsafe attachment mode [26] [27], the healthy growth of the adults, especially parents of children's personality have inescapable responsibility, good family and peer relations are also the important condition of children's personality development, in the absence of a good relationship, Trauma and PTSD related symptoms suffered by children will have an irreversible negative impact on their personality formation, and there are gender and age differences in the incidence of PTSD, and the conclusion that the neuroendocrine response caused by trauma leads to higher susceptibility of women than men in children has also been confirmed [28]. The incidence of PTSD was positively correlated with age, and children of different ages had different perception, understanding, response and memory of trauma. Typical AVOIDANCE symptoms of PTSD were more common in young children, while increased alertness and repeated experience were more common in older children.

\section{Treatment}

Treatment for PTSD includes individual, family, group, behavioral, and psychopharmacological interventions. Brief psychotherapy can produce definite cura- 
tive effects [29].

\subsection{Individual Therapy}

Approaches to individual therapy include play therapy, psychodynamic psychotherapy, anxiety management techniques, and interpretation of common posttraumatic symptoms and general course of illness. Older children and adolescents are suitable for direct therapy, while younger children are suitable for roleplaying and projective experiences (such as games, art, storytelling, etc.). Other literature has reported that desensitization [30], relaxation training, cognitive behavioral therapy [31] and other behavioral techniques are also beneficial to the treatment of children with PTSD. The key to treatment is to change the child's selfconcept from "victim" to "survivor". Reexperience symptoms and increased alertness can be induced in the treatment process, so it is necessary to fully understand the trauma experience of children, grasp the treatment schedule, so that children can integrate the trauma. It also needs to be pointed out that while treating the child victims, the individual treatment of the child perpetrators should not be neglected [32].

\subsection{Medication}

Medication can be used as an adjunct when the symptoms of PTSD are not easily relieved in children. Whether, what, and for how long depends on the specific symptoms and stage of the disease. Positive symptoms, such as repeated repetition and alertness, respond better to drugs than negative symptoms, such as avoidance. Comorbidities should also be considered when selecting drugs. Clonidine has been reported to be effective in the treatment of persistent alertness, especially the enhancement of the startle response and sleep disturbances. Propranolol has also been shown to help children with PTSD.

\subsection{Group Therapy}

When more children experience trauma, group therapy is often used. It enables the victim to recognize the symptoms and explain the course of the disease appropriately and with age. Sharing the experience with peers who have gone through the same or similar experience provides reassurance and an opportunity for satisfaction from helping others. Children who need intensive individual help can also be identified. Group therapy also has disadvantages. Not all children feel at ease in groups, and group therapy can potentially re-traumatize children through re-experiencing their own experiences or those of others. Children may adopt immature coping styles before fully examining their own reactions. The expression of anger and aggression can also cause anxiety among peers, so it must be restricted in time. If this occurs, individual treatment is required.

\subsection{Family Therapy}

The family plays an important role in children's adjustment to trauma. Avoidance and denial are often manifested in family therapy, in which parents can 
deny their children's pain to protect them. The parents' own trauma may be so intense that the child is neglected, perhaps because of the child's lack of language. The goal of family therapy is to help children regain a sense of security, normalize emotional responses and reduce secondary stress.

\subsection{Staged Intervention}

With the development of traumatized children, new problems related to trauma often appear, which will have a potential impact on future development. Therefore, staged intervention strategies should be timely planned after acute trauma intervention.

\subsection{School Intervention}

School is an appropriate environment for the rehabilitation of traumatized children, minimization of symptoms and adaptation to the child's developmental level, as well as a place where PTSD and its symptoms, such as intrusive associations and inattention, can easily affect academic performance and social adjustment. School interventions include: 1) classroom interventions for traumatic events and stress responses; 2) Opportunities to talk and discuss; 3) Group activities; 4) Projection technology; 5) Assess psychological responses formally and informally, correct false perceptions and fears, and promote recovery [33].

\section{Summary}

Although research on PTSD has been going on for many years, there is still little information about this particular group of children. The clinical manifestations of PTSD are also varied in children and adults due to their physical and mental immaturity, poor verbal expression, and poor tolerance to traumatic events. The diagnostic techniques and methods of PTSD in children are still immature, and the early psychological intervention is easy to be ignored. Clinical diagnosis techniques and methods for children with PTSD are still immature and early psychological intervention is easy to be neglected. Therefore, medical workers should focus on the early diagnosis, treatment, psychological intervention and individualized treatment of PTSD in children in the future research work. Only according to the characteristics of children with PTSD to find the best treatment, can we fundamentally eliminate the children's symptoms, so that they can reintegrate into the society.

\section{Foundation Project}

The paper is supported by Hainan Natural Science Foundation (Project No.820MS167).

\section{Conflicts of Interest}

The authors declare no conflicts of interest regarding the publication of this paper. 


\section{References}

[1] Guo, J.-C., Guo, M., Yang, Y.-J., Wang, X.-D., Juan, Y., Gao, Y.-S., Fu, L.-Q., Jiang, X.-L., Fu, L.-M. and Huang, T. (2018) Correlations of Four Genetic Single Nucleotide Polymorphisms in Brain-Derived Neurotrophic Factor with Posttraumatic Stress Disorder. Psychiatry Investigation, 15, 407-412. https://doi.org/10.30773/pi.2017.06.17.1

[2] Wang, M.Z., Liu, C.M. and Zhang, G.Q. (2018) Effects of Agomelatine on Memory Impairment and Hippocampal ERK5 Expression in Rats with Post-Traumatic Stress Disorder. Chinese Journal of Behavioral Medicine and Brain Science, 27, 971-975.

[3] Yan, S., Gao, S.L., Chen, Y.J., et al. (2016) Expression of Bax in Hippocampal Astrocytes of Rats with Post-Traumatic Stress Disorder. Journal of North China University of Science and Technology (Medical Edition), 18, 421-424.

[4] Guo, J.-C., Yang, Y.-J., Zheng, J.-F., Guo, M., et al. (2019) Functional rs6265 Poly- morphism in the BDNF Gene Confers Protection against Neurocognitive Dysfunction in Posttraumatic Stress Disorder among Chinese Patients with Hepatocellular Carci- noma. Journal of Cellular Biochemistry, 120, 10434-10443.

[5] Ericka, B.C., Anderson, J.L., Sharp, C., Langley, H.A. and Venta, A. (2021) Attachment Mentalization and Criterion B of the Alternative DSM-5 Model for Personality Disorders (AMPD). Borderline Personality Disorder and Emotion Dysregulation, 8, 23. https://doi.org/10.1186/s40479-021-00163-9

[6] Lang, X.Q., Lin, L., Ji, L., Yuan, X.Y., Chen, Q., Ran, Y.-M., Chen, H.-S., Li, L., Wang, J.-M., Wang, Z.-G., Gregersen, H., Zou, D.-W., Liang, H.-P. and Yang, M. (2017) P2X3 Receptor-Mediated Visceral Hyperalgesia and Neuronal Sensitization Following Exposure to PTSD-Like Stress in the Dorsal Root Ganglia of Rats. Neurogastroenterology Motility, 29, 138-140. https://doi.org/10.1111/nmo.12976

[7] George, S.A., Stout, S.A., Tan, M., Knox, D. and Liberzon, I. (2013) Early Handling Attenuates Enhancement of Glucocorticoid Receptors in the Prefrontal Cortex in an Animal Model of Post-Traumatic Stress Disorder. Biology of Mood \& Anxiety Disorders, 3, 22. https://doi.org/10.1186/2045-5380-3-22

[8] Guo, J.-C., Jiang, X.-L., Yang, Y.-J., Zheng, X.-A., Guo, M., et al. (2018) CpG Methylation of Brain-Derived the Neurotrophic Factor Gene Promoter as a Potent Diagnostic and Prognostic Biomarker for Post-Traumatic Stress Disorder. International Journal of Clinical and Experimental Pathology, 11, 5101-5109.

[9] Yuan, L. and Li, J.-Y. (2019) Exosomes in Parkinson's Disease: Current Perspectives and Future Challenges. ACS Chemical Neuroscience, 10, 964-972. https://doi.org/10.1021/acschemneuro.8b00469

[10] Ding, J.L., Han, F., Shi, Y.X., et al. (2015) Expression of PDE-4 and CREB in Amygdala of Rats with Post-Traumatic Stress Disorder. Journal of Histochemistry \& Cytochemistry, 24, 435-438.

[11] Saxena, M., Balaji, S.A., Deshpande, N., Ranganathan, S., Pillai, D.M., Hindupur, S.K., Rangarajan, A. (2018) AMP-Activated Protein Kinase Promotes Epithelial-Mesenchymal Transition in Cancer Cells through Twist1 Upregulation. Journal of Cell Science, 131. https://doi.org/10.1242/jcs.208314

[12] Geng, F., Liu, H. and Wang, H.T. (2014) Decreased Phosphorylation of Akt and mTOR in Amygdala Neurons of Rats with Post-Traumatic Stress Disorder. Journal of Chengde Medical College, 5, 382-384.

[13] Shemesh, E., Bartell, A. and Newcom, J. (2002) Assessment and Treatment of Depressive Disorders in Medically Ill Children. Current Psychiatry Reports (Current Sciences), 4, 88-92. https://doi.org/10.1007/s11920-002-0040-7 
[14] Henschel, A.V., Williams, J.L. and Hardt, M.M. (2021) The Role of Experiential Avoidance and Emotion Regulation in DSM-5 Posttraumatic Stress Symptomatology. Journal of Loss and Trauma, 26, 527-539. https://doi.org/10.1080/15325024.2020.1841506

[15] Gao, J.Q., Li, F., Liu, Y., et al. (2017) Protein Expression of GATl, KCC2, GAD67 and GABA in Different Brain Regions of Post-Traumatic Stress Disorder Rats. Jilin Traditional Chinese Medicine, 37, 171-176.

[16] North, C.S., Pfeferbaum, B., Tivis, L., et al. (2004) The Course of Posttraumatic Stress Disorder in a Follow-Up Study of Survivors of the Oklahoma City Bombing. Annals of Clinical Psychiatry, 16, 209-215. https://doi.org/10.1080/10401230490522034

[17] Karamustafalioglu, O.K., Zohar, J., et al. (2006) Natural Course of Post-Traumatic Stress Disorder. A 20-Month Prospective Study of Turkish Earthquake Survivors. Journal of Clinical Psychiatry, 67, 882-889. https://doi.org/10.4088/JCP.v67n0604

[18] Winston, F., Kassam-Adams, N., Garcia-Espa, F., Ittenbach, R. and Cnaan, A. (2003) Screening for Risk of Persistent Posttraumatic Stress in Injured Children and Their Parents. Journal of the American Medical Association, 290, 643-649. https://doi.org/10.1001/jama.290.5.643

[19] Stoddard, F.J. (2002) Care of Infants, Children and Adolescents with Bum Injuries. In: Lewis, M., Ed., Child and Adolescent Psychiatry, 3rd Edition, Lippincott Williams \& Wilkins, Philadelphia, 1188-1208.

[20] Tamowski, K.J., Rasnake, L.K., Gavaghan-Jones, M.P., et al. (1991) Psychosocial Sequelae of Pediatric Bum Injuries: A Review. Clinical Psychology Review, 11, 371-398. https://doi.org/10.1016/0272-7358(91)90114-A

[21] Meyer, W., Murphy, L., Bemiger, F., et al. (1999) The Sequence of Behavior Patterns of Children Burned before the Age of 4. Journal of Burn Care \& Rehabilitation, 20, S182.

[22] Johnson, J., Gorga, D., Silverberg, R., et al. (1998) The Relationship among the Physical, Developmental, and Functional Outcomes of Children (6 Months-6 Years Old) at one Month Post-Burn. Journal of Burn Care \& Rehabilitation, 19, S152. https://doi.org/10.1097/00004630-199801001-00035

[23] Saxe, G., Stoddard, F., Hall, E., et al. (2005) Pathways to PTSD I: Children with Bums. American Journal of Psychiatry, 167, 1299-1304. https://doi.org/10.1176/appi.ajp.162.7.1299

[24] Stuber, M.L., Kazak, A.E., Meeske, K., et al. (1997) Predictors of 125 PTSD in Childhood Cancer Survivors. Pediatrics, 100, 958-964. https://doi.org/10.1542/peds.100.6.958

[25] Winje, D. and Ulvik, A. (1998) Long-Term Outcome of Trauma in Children. The Psychological Consequences of a Bus Accident. Journal of Child Psychology and Psychiatry, 39, 635-642. https://doi.org/10.1017/S0021963098002479

[26] Kolts, R.L., Robinson, A.M. and Tracy, J.J. (2004) The Relationship of Sociotropy and Autonomy to Posttraumatic Cognitions and PTSD Symptomatology in Trauma Survivors. Journal of Clinical Psychology, 60, 53-63. https://doi.org/10.1002/jclp.10193

[27] Chou, C.C., Lee, K.H., Lai, I.L., Wang, D., Mo, X., Kulp, S.K., Shapiro, C.L. and Chen, C.S. (2018) AMPK Reverses the Mesenchymal Phenotype of Cancer Cells by Targeting the Akt-MDM2-Foxo3a Signaling Axis. Cancer Research, 78, 3401. https://doi.org/10.1158/0008-5472.CAN-18-0559

[28] Wamser-Nanney, R. and Cherry, K.E. (2018) Children's Trauma-Related Symptoms Following Complex Trauma Exposure Evidence of Gender Differences. Child Abuse \& Neglect, 77, 188-197. https://doi.org/10.1016/j.chiabu.2018.01.009

[29] Goenjian, A.K., Karayan, I., Pynoos, R.S., et al. (1997) Outcome of Psychotherapy 
among Early Adolescents after Trauma. American Journal of Psychiatry, 154, 536-542. https://doi.org/10.1176/ajp.154.4.536

[30] Scheck, M.M., et al. (1998) Brief Psychological Intervention with Traumatized Young Women: The Efficacy of Eye Movement Desensitization and Reprocessing. Journal of Traumatic Stress, 8, 25-44. https://doi.org/10.1023/A:1024400931106

[31] Farrell, S.P., Hains, A.A. and Davies, W.H. (1998) Cognitive Behavioral Interventions for Sexually Abused Children Exhibiting PTSD Symptomatology. Behavior Therapy, 29, 241-255. https://doi.org/10.1016/S0005-7894(98)80005-1

[32] March, J.S., Amaya-Jackson, L., Murray, M.C., et al. (1998) Cognitive-Behavioral Psychotherapy for Children and Adolescents with Posttraumatic Stress Disorder after a Single-Incident Stressor. The Journal of the American Academy of Child and Adolescent Psychiatry, 371, 585-593.

https://doi.org/10.1097/00004583-199806000-00008

[33] Schweitzer, M.G. and Puig-Verges, N. (1998) Preliminaries to the Treatment of Psychic Distress States in Children in Exceptional Situations. Annales Médico-Psychologiques, $156,10-13$. 